\title{
ANALYTICAL DESIGN OF DIGITAL NONRECURSIVE MAXIMALLY FLAT FRACTIONAL HILBERT TRANSFORMER
}

\author{
Soo-Chang Pei and Peng-Hua Wang \\ Department of Electrical Engineering, National Taiwan University, Taipei, Taiwan \\ Email address: pei@cc.ee.ntu.edu.tw
}

\begin{abstract}
Recently, a generalization of the Hilbert transformer, the fractional Hilbert transformer, was defined and developed. In this paper, we propose a design of the FIR filter to realize the fractional Hilbert transformer based on the maximally flat approach. The impulse response is obtained explicitly. An efficient realization structure is constructed according to some properties of the impulse response.
\end{abstract}

\section{INTRODUCTION}

Hilbert transform (HT) is a basic and important tool in signal processing. In a communication system, it is used for single side-band modulation and then reduces the bandwidth needed for transmission. HT is also used for edge detection [1].

In [2], the HT is generalized into the fractional Hilbert transform (FHT) and two alternative definitions are given. One is a modification of the definition of HT and the frequency response is expressed by

$$
H_{f h t}(\omega)= \begin{cases}e^{-j \alpha \pi / 2} & \text { for } \omega>0 \\ e^{j \alpha \pi / 2} & \text { for } \omega<0\end{cases}
$$

Note that the special case of $\alpha=1$ leads the frequency response of HT. Another is by using the above definition as well as the Fractional Fourier transform to achieve a twoparameter FHT system. The discrete version of the FHT is developed and proposed in [3].

Both FIR and IIR filters are investigated to realize the HT in extensive literature. In [4] and [5], the impulse responses of the FIR HT are analytically obtained in the maximally flat (MF) sense. A realization scheme based on decomposing the transfer function of HT into allpass subfilters is proposed in [6].

In this paper, we design a FIR filter with frequency response expressed by Eq. (1). This ideal frequency response is modified to obtain a causal filter. The design method is based on the MF approach by which the designed filter has a very flat response in the midband frequencies.

\section{DESIGN OF MAXIMALLY FLAT FHT}

The transfer function of a causal FIR filter of order $N$ is characterized by

$$
H(z)=\sum_{n=0}^{N} h(n) z^{-n}
$$

where $h(n)$ is the impulse response to be design. Evaluating the above equation along the unit circle, the frequency response is obtained as

$$
H\left(e^{\jmath \omega}\right)=\sum_{n=0}^{N} h(n) e^{-j n \omega} .
$$

If the desired frequency response is even or odd about the DC frequency $(\omega=0), h(n)$ may exhibits the symmetry and then the Eqs. (2) and (3) can be simplified. However, since Eq. (1) is neither even nor odd, we can not specify any symmetric property to $h(n)$ a priori.

In order to design a causal FHT, the ideal frequency response expressed in Eq. (1) must be modified because it lacks of a delay and can not be realized in practice. For the $N$ thorder FIR system to be designed, we put an additional delay term $e^{-j N \omega / 2}$ into Eq. (1) and obtain the desired frequency response as

$$
H_{d}(\omega)= \begin{cases}e^{-j(\alpha \pi / 2+N \omega / 2)} & \text { for } \omega>0 \\ e^{j(\alpha \pi / 2-N \omega / 2)} & \text { for } \omega<0\end{cases}
$$

In this paper, we consider the case of odd $N$ and result in an even length FIR filter.

To design a FIR filter in the MF sense, we equate the derivatives of the desired frequency response in Eq. (4) with those of the frequency response in Eq. (3) up to a suitable order, i.e.,

$$
\sum_{n=0}^{N} h(n) n^{k} e^{-j n \omega}=\left(\frac{N}{2}\right)^{k} e^{-j(\alpha \pi / 2+N \omega / 2)}
$$

for $\omega>0$ and

$$
\sum_{n=0}^{N} h(n) n^{k} e^{-j n \omega}=\left(\frac{N}{2}\right)^{k} e^{j(\alpha \pi / 2-N \omega / 2)}
$$


for $\omega<0$ where $k=0,1, \ldots, K$ and $K$ is determined by the number of impulse response.

For the MF design, the next step is to choose the frequencies at which the MF conditions expressed by the above equations are satisfied. In this paper, the MF frequencies of $\pm \pi / 2$ are chosen to approximate the desired frequency response. Substituting Eqs. (5) and (6) with $\omega=\pi / 2$ and $\omega=-\pi / 2$ respectively, these equations become

$$
\begin{aligned}
\sum_{n=0}^{N} h(n) n^{k} e^{-j n \pi / 2} & =\left(\frac{N}{2}\right)^{k} e^{-j \phi} \\
\sum_{n=0}^{N} h(n) n^{k} e^{j n \pi / 2} & =\left(\frac{N}{2}\right)^{k} e^{j \phi}
\end{aligned}
$$

where $\phi=\alpha \pi / 2+N \pi / 4$ for short.

In order to determine the largest allowable $k$ in the above equations, the number of these equations is examined. For $k$ th derivative, two MF conditions must be satisfied since the MF frequencies are specified to be $\pi / 2$ and $-\pi / 2$. That is, there are two equations constructed for each choice of $k$. To solve the linear equations with $N+1$ unknowns expressed by Eqs. (7) and (8), $k$ is equal to $(N-1) / 2$ at most. Hence there are $N+1$ linear equations used for solving the impulse response of the MF FHT in Eqs. (7) and (8).

To solve these equations analytically, they are simplified further. For each $k$, by adding Eq. (7) and Eq. (8) and by subtracting Eq. (7) from Eq. (8), we deduce

$$
\begin{aligned}
\sum_{n=0}^{N} h(n) n^{k} \cos \left(\frac{n \pi}{2}\right) & =\left(\frac{N}{2}\right)^{k} \cos \phi \\
\sum_{n=0}^{N} h(n) n^{k} \sin \left(\frac{n \pi}{2}\right) & =\left(\frac{N}{2}\right)^{k} \sin \phi
\end{aligned}
$$

for $k=0,1, \ldots,(N-1) / 2$. Since $\cos (n \pi / 2)=0$ and $\sin (n \pi / 2)=0$ for odd $n$ and even $n$ respectively, the above equations are actually in the forms

$$
\begin{gathered}
\sum_{n=0}^{(N-1) / 2} h(2 n)(-1)^{n}(2 n)^{k}=\left(\frac{N}{2}\right)^{k} \cos \phi \\
\sum_{n=0}^{(N-1) / 2} h(2 n+1)(-1)^{n}(2 n+1)^{k}=\left(\frac{N}{2}\right)^{k} \sin \phi
\end{gathered}
$$

for $k=0,1, \ldots,(N-1) / 2$. Because both of the Eqs. (11) and (12) describe $(N+1) / 2$ equations in $(N+1) / 2$ unknowns, these two sets of equations can be solved directly. Accordingly, the coefficients of the even numbered impulse and of the odd numbered one are solved separately. Carrying out the Cramer's rule [7] and noting the involved Vandermonde determinants, after some algebraic manipulations, the solutions are obtained explicitly by

$$
\begin{aligned}
h(2 n) & =\frac{k_{e} \cos \phi}{\left(n-\frac{N}{4}\right)\left(\frac{N-1}{2}-n\right) ! n !} \\
h(2 n+1) & =\frac{k_{o} \sin \phi}{\left(n-\frac{N-2}{4}\right)\left(\frac{N-1}{2}-n\right) ! n !}
\end{aligned}
$$

where

$$
\begin{aligned}
& k_{e}=\prod_{i=0}^{(N-1) / 2}\left(i-\frac{N}{4}\right) \\
& k_{o}=\prod_{i=0}^{(N-1) / 2}\left(i-\frac{N-2}{4}\right)
\end{aligned}
$$

Therefore, the impulse response of the MF FHT is solved analytically.

It is easy shown that the impulse responses expressed by Eqs.(13) and (14) are neither symmetric nor antisymmetric, i.e., $h(n) \neq \pm h(N-n)$, in general, since the irrational factor $\cos \phi$ is not equal to $\sin \phi$. Consequently, $N+1$ multiplications per input sample are required in comparison with $(N+1) / 2$ multiplications for linear phase FIR filters of order $N$. In next section, we propose an efficient realization scheme of about $(N+1) / 2$ multiplications by exploiting the detailed properties of the calculated impulse response.

\section{REALIZATION OF MAXIMALLY FLAT FHT}

The impulse response exhibits fine symmetry. Let the normalized impulse response be $\tilde{h}(2 n)=h(2 n) / \cos \phi$ and $\tilde{h}(2 n+1)=h(2 n+1) / \sin \phi$. Then the transfer function expressed by Eq. (2) is represented as

$$
H(z)=\cos \phi H_{e}(z)+\sin \phi z^{-1} H_{o}(z)
$$

where

$$
\begin{aligned}
& H_{e}(z)=\sum_{n=0}^{(N-1) / 2} \tilde{h}(2 n) z^{-2 n} \\
& H_{o}(z)=\sum_{n=0}^{(N-1) / 2} \tilde{h}(2 n+1) z^{-2 n} .
\end{aligned}
$$

The first property of the impulse response is that the factor $k_{o}$ is equal to $k_{e}$ without regard to their signum. This fact is obtained by changing the multiplication order in Eq. (16) and explicitly expressed as

$$
k_{o}=(-1)^{(N+1) / 2} k_{e} .
$$

By means of the above equality, we deduce that

$$
\tilde{h}(N-2 n)=(-1)^{(N-1) / 2} \tilde{h}(2 n)
$$


for $n=0,1, \ldots,(N-1) / 2$. Note that $\tilde{h}(N-2 n)=$ $\tilde{h}\left[2\left(\frac{N-1}{2}-n\right)+1\right]$, i.e., $\tilde{h}(N-2 n)$ is an odd numbered coefficient while $\tilde{h}(2 n)$ is an even numbered one.

The key equation (21) implies the important property of $H_{o}(z)$ being the mirror-image polynomial about $H_{e}(z)$, i.e.,

$$
H_{o}(z)=(-1)^{(N-1) / 2} z^{-(N-1)} H_{e}\left(z^{-1}\right) .
$$

Based on this symmetric property, an efficient realization structure is designed. We begin with the fact that any FIR filter can be expressed as a sum of a symmetric and an antisymmetric FIR filter. Accordingly $H_{e}(z)$ is decomposed as

$$
H_{e}(z)=H_{s}(z)+H_{a}(z)
$$

where

$$
\begin{aligned}
& H_{s}(z)=\frac{1}{2}\left[H_{e}(z)+z^{-(N-1)} H_{e}\left(z^{-1}\right)\right] \\
& H_{a}(z)=\frac{1}{2}\left[H_{e}(z)-z^{-(N-1)} H_{e}\left(z^{-1}\right)\right] .
\end{aligned}
$$

Note that $H_{s}(z)$ represent a symmetric FIR filter and $H_{a}(z)$ represents a antisymmetric one. According to the above equations and Eq. (22), we have

$$
H_{o}(z)=(-1)^{\frac{N-1}{2}}\left[H_{s}(z)-H_{a}(z)\right] .
$$

Substituting Eq. (17) with Eqs. (23) and (26), the transfer function of proposed MF FHT is expressed as a linear combination of $H_{s}(z)$ and $H_{a}(z)$

$$
\begin{aligned}
H(z) & =\cos \phi\left[H_{s}(z)+H_{a}(z)\right] \\
& +(-1)^{\frac{N-1}{2}} \sin \phi z^{-1}\left[H_{s}(z)-H_{a}(z)\right] .
\end{aligned}
$$

This structure is shown in Fig. 1.

To evaluate the number of multiplications expressed by Eq. (27), note that the coefficients of the odd numbered impulse response are zeros in $H_{s}(z)$ and in $H_{a}(z)$. Since they are linear phase FIR filters of $(N-1)$ th order, each of them has $(N+1) / 2$ nonzero coefficients and only halves are necessary for realization. We conclude that the number of multiplications for realizing the MF FHT shown in Fig. 1 is $(N+5) / 2$. This number is two more than that in the common linear phase FIR filter for the factors $\sin \phi$ and $\cos \phi$.

\section{DESIGN EXAMPLES}

In this section, we demonstrate some design examples of the proposed MF FHT for various design parameters. The MF FHT is applied to a rectangular function. This can be regarded as an example of the 1-D edge detection.

Example 1. In this example, a set of MF FHT of 15th order are designed. The fractional factors $\alpha$ are $0.25,0.5,0.75$, and 1 for each filter. Figs. $2 a$ and $2 b$ show the magnitude and phase responses respectively. The phase responses are normalized to remove the linear component in the following manner

$$
\tilde{\theta}(\omega)=\theta(\omega)+\frac{N \omega}{2}
$$

where $\bar{\theta}(\omega)$ and $\theta(\omega)$ are the normalized phase response and the actual phase response, respectively. $N$ is the filter order and equal to 15 in this example.

Example 2. The filters of different orders are designed with $\alpha=0.5$ in this example. The orders are $15,17,19$, and 21. Fig. 3 a shows the magnitude responses and Fig. 3 b shows the normalized phase responses. Note that the obvious gap between the curves of $N=15$ and $N=19$ and those of $N=17$ and $N=21$.

Example 3. The proposed MF FHT is used for filtering a testing signal. This input signal is a discrete rectangular function which is given by

$$
x(n)= \begin{cases}1 & \text { for } 108 \leq n \leq 147 \\ 0 & \text { for } 0 \leq n \leq 107 \text { or } 148 \leq n \leq 255 .\end{cases}
$$

That is, The width of $x(n)$ is equal to 40 points and its length is 256 points. This signal is also used for demonstration in [2] and [3]. The order of the FIR filter is equal to 15. Fig. 4 shows the the magnitudes of the ideal FHT outputs of $x(n)$. The ideal outputs are calculated by the discrete FHT described in [3]. The testing signal is filtered by the MF FHT and the magnitudes of the resulting outputs are shown in Fig. 5. The rising edges are emphasized for $0<\alpha<1$ , whereas the falling edges are emphasized for $1<\alpha<2$. If $\alpha=1$, both types of edges are equally emphasized. Note that the overshoots occur at the edges for $\alpha=0$.

\section{CONCLUSIONS}

In this paper, the design of the nonrecursive MF FHT of odd order is proposed. The impulse response is obtained explicitly. Based on symmetric properties of the impulse response, an efficient realization structure is described. The resulting complexity is approximately equal to that of a linear phase FIR filter of the same order.

\section{REFERENCE}

[1] K. Kohlmann, "Corner detection in natural images based on the 2-D Hilbert transform," Signal Processing, vol. 48, pp. 225-234, 1996.

[2] A. W. Lohmann, D. Mendlovic and Z. Zalevsky, "Fractional Hilbert transform," Optics Letters, vol. 21, no. 4, pp. 281-283, February 1996.

[3] S.-C. Pei and M.-H Yeh, "Discrete Fractional Hilbert Transform," Proc. IEEE Int. Symposium on Circuits and Systems, Monterey, California, May 1998. 
[4] T. Cooklev and A. Nishihara, "Maximally Flat FIR Hilbert Transformer," Int. J. of Circuit Theory Applicat., vol. 21, pp. 353-570, 1993.

[5] J. Le Bihan, "Coefficients of FIR Digital Differentiators and Hilbert Transformers for Midband Frequencies," IEEE Trans. Circuits and Systems-II, vol. 43, no. 3, pp. 272-274, March 1996.

[6] H. Johansson and L. Wanhammar, "Digital Hilbert Transformers Composed of Identical Allpass Subfilters," Proc. IEEE Int. Symposium on Circuits and Systems, Monterey, California, May 1998.

[7] B. Noble and J. W. Daniel, Applied Linear Algebra, Prentice-Hall, Inc. 1988.

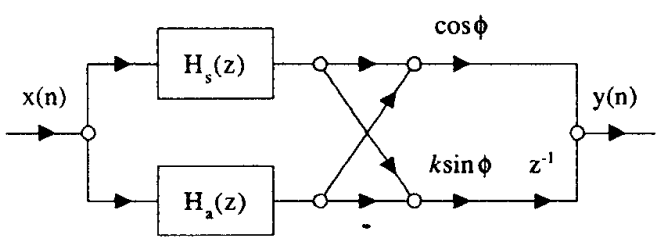

Figure 1: Block diagram of the proposed MF FHT. The factor $k$ in the lower branch is equal to $(-1)^{(N-1) / 2}$.

(a)

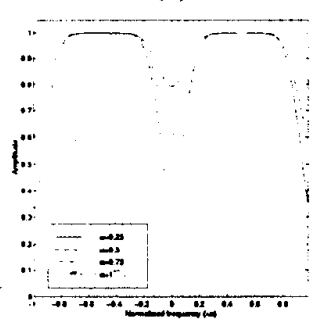

Figure 2: Frequency responses of 15 th order FIR MF FHT with various values of $\alpha=0.25,0.5,0.75$, and 1. (a) Magnitude responses. (b) Normalized phase responses. (a)

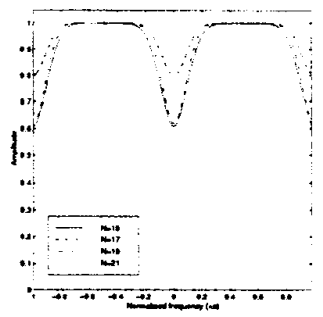

(b)

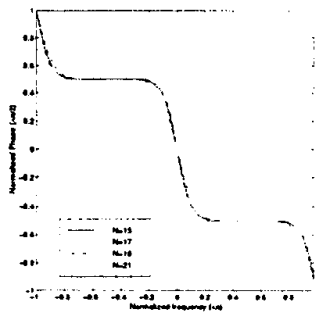

Figure 3: Frequency responses of FIR MF FHT of various orders with $\alpha=0.5$. (a) Magnitude responses. (b) Normalized phase responses.

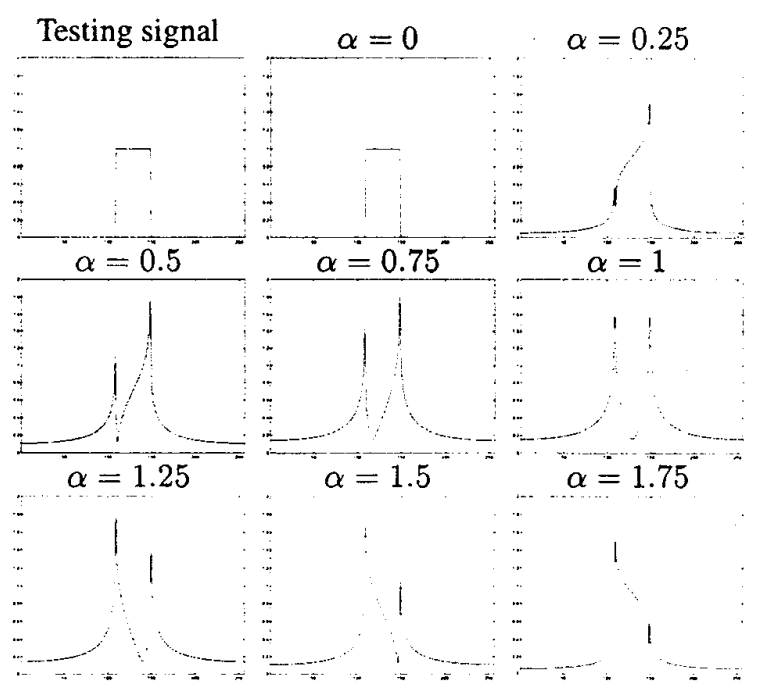

Figure 4: FHTs of the discrete rect function for various values of $\alpha$.

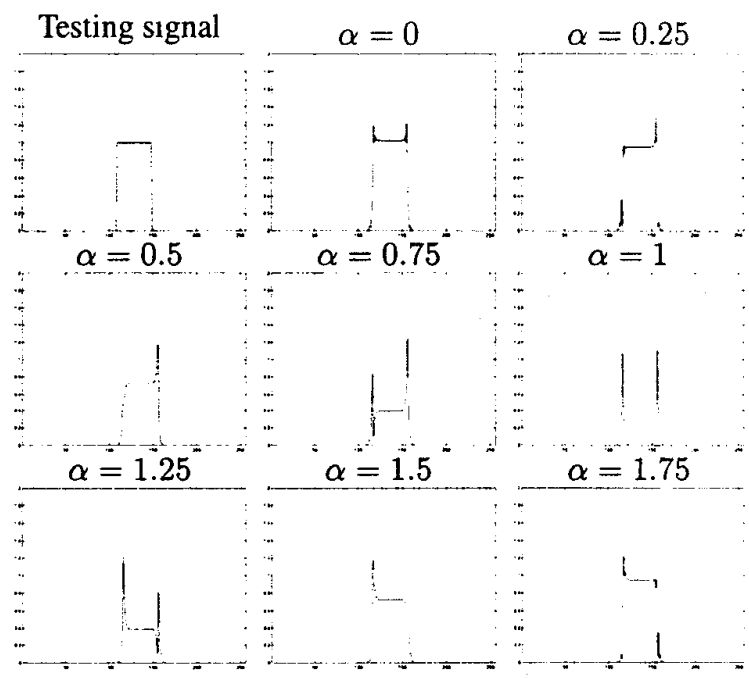

Figure 5: The MF FHT outputs of the discrete rect function for various values of $\alpha$. 